Institut für Veterinärbakteriologie

der Vetsuisse-Fakultät Universität Zürich

Direktor: Prof. Dr. M. M. Wittenbrink

Arbeit unter Leitung von Prof. Dr. med. vet. R. Hoop

\title{
Chlamydiae and Atherosclerosis: Can Psittacine Cases Support the Link?
}

\author{
Inaugural-Dissertation \\ zur Erlangung der Doktorwürde der \\ Vetsuisse-Fakultät Universität Zürich
}

\author{
vorgelegt von \\ Olivier Andreas Schenker \\ Tierarzt \\ von Walterswil SO \\ genehmigt auf Antrag von \\ Prof. Dr. R. Hoop, Referent \\ Prof. Dr. A. Pospischil, Korreferent
}

Zürich 2006 
Meinen Eltern 
Chlamydiae and atherosclerosis in pet birds

\title{
Chlamydiae and Atherosclerosis: Can Psittacine Cases Support the Link?
}

\author{
Olivier A. Schenker ${ }^{\mathrm{A}}$ and Richard K. Hoop
}

Institute of Veterinary Bacteriology, National Reference Center for Poultry and Rabbit Diseases, Vetsuisse-Faculty, University of Zurich, Winterthurerstrasse 270, 8057 Zurich, Switzerland

${ }^{A}$ Corresponding author. Email: olivier.schenker@vetbakt.unizh.ch 
SUMMARY. Atherosclerosis is a common disease in pet birds, particularly in psittacines. Little is known about the role of risk factors predisposing birds to this disease. In our study we tried to detect chlamydiae in formalin-fixed and paraffin-embedded atherosclerotic tissue from 103 pet birds in order to clarify their role in atherosclerosis. Methods used were PCR, sequencing and immunohistochemistry. Histopathologic examination served to classify the extent of atherosclerotic lesions. In the PCR, 4 (3.9\%) of 103 cases - all of them with advanced stages of atherosclerosis - were positive. Subsequent sequence analysis revealed high identities (94-100\%) with Chlamydophila psittaci in 3 cases. Interestingly, 2 of these birds came from Chlamydophila psittaci-infected populations. Due to the low incidence (3.9\%), the occurrence only in advanced stages and the association with Chlamydophila psittaci-infected avian populations a causal relationship between chlamydiae and atherosclerosis in pet birds is rather improbable.

Key words: Chlamydia, atherosclerosis, pet bird, PCR, immunohistochemistry

Abbreviations: $C .=$ Chlamydia Cp. $=$ Chlamydophila $;$ ELISA $=$ enzyme-linked immunosorbent assay; $\mathrm{IHC}=$ immunohistochemistry; $\mathrm{PCR}=$ polymerase chain reaction 


\section{INTRODUCTION}

Atherosclerosis is a slow and progressive disease that usually affects the large and medium sized arteries. As a result of thickening of the artery wall the diameter of the lumen decreases and the blood flow is reduced. In severe cases ischemia in the area receiving blood from the artery occurs.

Atherosclerosis is a principal cause of morbidity and mortality in humans and occurs spontaneously in other mammals and in birds (19). In man risk factors for this cardiovascular disease are among others elevated plasma cholesterol level, hypertension, exposure to cigarette smoking, increasing age, obesity and diabetes mellitus. There are also several studies that have addressed a possible role of infectious agents, such as viruses and bacteria, in atherogenesis $(9,14)$.

In humans, Chlamydophila pneumoniae has been most strongly associated with cardiovascular diseases. Some authors conclude that $C p$. pneumoniae is a possible etiological agent of atherosclerosis, while others deny it $(2,16,17)$.

In birds, the herpesvirus of Marek`s disease was shown to induce atherosclerosis in chickens $(12,13)$.

Atherosclerosis is also a common disease in birds, especially in orders such as Anseriformes (swan, geese, duck), Columbiformes (pigeons, doves) and Psittaciformes (parrots, parakeets). Within the last order the parrots are most susceptible to atherosclerosis, e.g. the African grey parrot and the amazons $(3,4,19)$. Atherosclerotic changes are mainly located in the thoracic part of the aorta and the brachiocephalic arteries. Clinical signs resulting from atherosclerosis are rare. Thus atherosclerosis is usually observed as an unexpected lesion at necropsy $(4,19)$. The role of risk factors predisposing birds to atherosclerosis is unknown, but since the lesions in birds closely resemble those in man, risk factors in humans may extend to birds (4). The intention of our retrospective study was to detect chlamydiae in formalin-fixed atherosclerotic tissue from birds, in order to clarify their role in atherosclerosis. 


\section{MATERIAL AND METHODS}

\section{Selection of cases}

Formalin(4\%)-fixed and paraffin-embedded tissue specimens from 103 pet birds necropsied between 1991 and 2005 at the National Reference Centre for Poultry and Rabbit Diseases (NRGK), University of Zurich, showing macroscopic atherosclerosis were investigated. Tissue specimens were collected from the heart and/or the main-stem vessels.

\section{Histopathology}

Histologic sections of all selected cases were stained with the hematoxylin and eosin (HE) technique and reviewed histologically to verify the presence of atherosclerosis. The extent of atherosclerosis was classified in 4 grades (Fig. 3): grade $1=$ slight atherosclerosis with fragmentation of the elastic fibres and increase of the extracellular matrix in the intima and media, grade $2=$ moderate atherosclerosis with additional accumulation of lipids in the intima, grade $3=$ development of plaques consisting of lipids and cholesterol clefts, sporadic cartilage and calcification, grade $4=$ severe atherosclerosis with intense and irregular thickening of the arterial wall with plaques containing more cartilage and calcification than those of grade 3 .

\section{DNA extraction for PCR screening}

From each paraffin block $30 \mu \mathrm{m}$ sections were cut and placed in sterile microcentrifuge tubes. Then $1 \mathrm{ml}$ xylene was added to remove the paraffin. After centrifugation at $15,000 \mathrm{rpm}$ for 5 min, the tissue pellet was extracted twice with absolute ethanol followed by centrifugation $(15,000 \mathrm{rpm}$; $5 \mathrm{~min})$ to remove remaining xylene, dried at $42 \mathrm{C}$ for $30 \mathrm{~min}$ and resuspended in $200 \mu l$ extraction buffer (containing $0.05 \%$ Tween 20, 1mM EDTA, 50mM Tris-HCl pH 8.5). After addition of $2 \mu 10.2 \mathrm{mg} / \mathrm{ml}$ proteinase $\mathrm{K}$, samples were incubated at $55 \mathrm{C}$ over night. Then proteinase $\mathrm{K}$ was inactivated by heating for $10 \mathrm{~min}$ at $100 \mathrm{C}$. DNA was purified with the phenol-chloroform-isoamylethanol method according to a standard protocol (Sambrook et al, 1989). Briefly samples were mixed twice with $200 \mu 1$ phenol with following incubation for 20 
min on ice and centrifugation at $10,000 \mathrm{rpm}$ for $5 \mathrm{~min}$. Subsequently the water phase was transferred to a clean tube and treated twice with IAC (chloroform-isoamylethanol 24:1) with following centrifugation at 10,000rpm for $5 \mathrm{~min}$. Afterward DNA was precipitated with $20 \mu \mathrm{l}$ sodium acetate and $400 \mu \mathrm{l}$ absolute ethanol for $30 \mathrm{~min}$ at $-80 \mathrm{C}$. Next samples were centrifuged at $13,000 \mathrm{rpm}$ for $30 \mathrm{~min}$ at $4 \mathrm{C}$ and DNA was washed with $200 \mu \mathrm{l}$ ethanol $70 \%$. After another centrifugation for 15 min the pellet was air-dried and resuspended in $20 \mu \mathrm{l}$ Nuclease-free water (Promega,WI USA).

\section{PCR detection of chlamydial DNA}

DNA extracted from formalin-fixed and paraffin-embedded tissue samples was screened for the presence of chlamydial DNA. Due to possible DNA degradation the primer pair of 23SQAPF2 (5' -GAACCTGAAACCA(AG)TAGC-3') and 23SAPR (5'CTGGCTCATCATGCAAAAGG-3') was applied to amplify a small 92-bp fragment of the $23 \mathrm{~S}$ rRNA gene. PCR was done in $25 \mu 1$ reaction mixture volumes consisting of $20 \mathrm{pmol}$ of each primer, 12.5 $\mu \mathrm{l}$ of HotStarTaq Master Mix from Qiagen (containing HotStarTaq DNA polymerase, PCR buffer with $1.5 \mathrm{mM} \mathrm{MgCl}_{2}$ and $200 \mu \mathrm{M}$ each $\mathrm{dNTP}$ ) and $1 \mu 1$ of each DNA template. The thermal cycling conditions were $95 \mathrm{C}$ for $15 \mathrm{~min}, 45$ cycles of denaturation at $94 \mathrm{C}$ for $30 \mathrm{~s}$, primer annealing at $50 \mathrm{C}$ for $30 \mathrm{~s}$, and elongation at $72 \mathrm{C}$ for $30 \mathrm{~s}$, and a final elongation at $72 \mathrm{C}$ for $5 \mathrm{~min}$ (24). Each run contained a negative control with Nuclease-free water substituting the template DNA, and a positive control obtained from formalin-fixed and paraffin-embedded tissues containing chlamydial DNA (either from birds or gnotobiotics piglets). $10 \mu 1$ of each PCR product were analysed by electrophoresis on an ethidium bromidestained 2\% agarose gel (Agarose Standard, Eurobio) in the presence of a 100bp ladder. PCR bands on the gel were visualized by UV illumination with a computer-aided bioimage system (Bio-Capt, LTF, Wasserburg, Germany). 


\section{Sequencing}

Of each PCR product $60 \mu 1$ was electrophoresed on an ethidium bromide-stained $2 \%$ agarose gel (Agarose Standard, Eurobio). Desired bands of amplicons were cut out under UV illumination and DNA was extracted using the MinElute Gel Extraction Kit from Qiagen. Samples were sent to Microsynth AG (Balgach, Switzerland) for direct sequencing. The obtained sequences were analyzed through BLAST search (http://www.ncbi.nlm.nih.gov/BLAST/).

\section{Immunohistochemistry}

Paraffin sections showing a suspect band in the PCR were processed for immunohistochemistry by using mouse monoclonal antibodies against chlamydial lipopolysaccharide (clone ACI-P) and Dako EnVision anti-mouse (K 4001). First sections were pretreated by microwave heating for $20 \mathrm{~min}$ in citrate buffer (Dako S2031) for antigen retrieval. Then they were incubated with the primary antibody at a working dilution of 1:200 (Dako S2022) for $60 \mathrm{~min}$ at room temperature. After incubation for $30 \mathrm{~min}$ with the second antibody at room temperature, sections were developed in peroxidase/3-amino-gethylcarbazole (AEC) substrate solution for $10 \mathrm{~min}$ at room temperature, counterstained with hematoxylin and coverslipped. From each section a negative control was performed using the antibody diluent (Dako S2022) without the primary antibody. As a positive control intestinal tissues from gnotobiotic piglets experimentally infected with Chlamydia suis were used. 


\section{RESULTS}

\section{Selection of cases}

The 103 birds consisted of the following avian orders: 92 Psittaciformes, 3 Passeriformes, 2 Anseriformes, 2 Ciconiiformes and 4 birds from other avian orders. In the order Psittaciformes, African grey parrots $(n=31)$ and amazons $(n=21)$ had the highest incidences, with $30.3 \%$ respectively $20.4 \%$ (Figure 2). The age of the birds ranged from 2 to 78 years (Table 1).

\section{Histopathology}

Histopathologic examination revealed in all cases slight to severe atherosclerosis. 15 of the cases were classified grade 1, 20 were rated grade 2, 24 were classified grade 3, and in 40 cases a severe atherosclerosis with grade 4 was observed. No relation between age of bird and degree of atherosclerosis was found (Fig. 1), but there was an association between species and severity of atherosclerosis (Fig.2).

\section{PCR}

Of all 103 samples tested, 22 (21.3\%) were PCR suspect, showing a band at $50-200 \mathrm{bp}$. For further confirmation $30 \mu \mathrm{m}$ sections from these 22 specimens were cut again and retested with the same method and in the same run, using more positive controls. A strong positive band at 92bp was seen in 4 specimens, resulting in a total rate of $3.9 \%(n=103)$. Positive and negative controls reacted appropriately.

\section{Sequencing}

Online BLAST searches revealed for all 4 PCR-positive samples applied for direct sequencing similarities with sequences of the genus Chlamydophila. In 3 cases high identities 
(94-100\%) with $C p$. psittaci were found, whereas in one case no further specification was possible. Results of the PCR positive cases are summarized in Table 2.

\section{Immunohistochemistry}

A positive staining was seen in 9 of the 22 (40.9\%) PCR-suspect sections prepared for immunohistochemistry. Granules with positive staining were found mainly at locations of the vessel wall, where the atherosclerotic alterations were severe. In the extracellular matrix as well as in the cytoplasm of macrophages and also of smooth muscle cells immunopositive signals were observed.

In negative control sections immunopositive staining was found at the same locations as in the 9 sample sections. 


\section{DISCUSSION}

Chlamydiae are unique intracellular pathogens with a biphasic developmental cycle consisting of the infectious elementary body (EB) and the non-infectious reticulate body (RB) (10). Until 1999 the order contained only one genus, Chlamydia, with 4 recognized species, namely $C$. psittaci, C.trachomatis, C. pneumoniae and C. pecorum (11). Recently the order has been reclassified on the basis of taxonomic analysis of the $16 \mathrm{~S}$ and $23 \mathrm{~S}$ rRNA genes. Three new families, Parachlamydiaceae, Simkaniaceae and Waddliaceae were proposed, while the family Chlamydiaceae was divided into two genera, Chlamydia and Chlamydophila, with a total of nine species (11). Chlamydiae cause a variety of diseases in different animal species including humans, birds, koalas, cats, pigs, cattle, sheep and goats (10).

Chlamydophila pneumoniae was first described in humans in 1986. It is a common cause of upper respiratory tract infections and accounts for up to $10 \%$ of community acquired pneumonia (15). Recently it has been associated with several chronic diseases such as Alzheimer's disease and atherosclerosis. The first report that discussed a possible connection between $C p$. pneumoniae and atherosclerosis was a serologic study performed in Finland in 1988 (22). Seroepidemiologic studies were followed by studies in which the organism was identified in atherosclerotic tissue by electron microscopy, immunocytic chemical staining and PCR $(5,6,8,23)$. However, the results of those studies are inconsistently reporting a large variability in detection rates (6). Therefore the real prevalence of $C p$. pneumoniae within atherosclerotic lesions and its role in atherogenesis is still a matter of controversial debate in human medicine.

Atherosclerosis in birds - mainly in parrots - seems to be more prevalent and severe than in

any species of mammals, except for men (4). Postmortem findings in birds examined between 1991 and 1997 revealed an incidence for atherosclerosis of $7 \%$ for the psittacines, whereas only $0.6 \%$ of the passerines showed this disease. Of the atherosclerotic psittacines, $79 \%$ were parrots, with the highest incidences for the African grey parrot (35\%) and the amazons (22\%) (1). In contrast to man, little is known about risk factors of atherosclerosis in birds. Age, genetics, plasma cholesterol levels, diet, inactivity, social stress and obesity were discussed in parrots (4).

Cp. pneumoniae has yet never been found in birds. In a recent study from Sudler et al, all fifty-one chlamydial isolates from birds collected in Switzerland were classified as $C p$. psittaci (25). On the other hand $C p$. psittaci is widespread in avian populations, where it causes disease with varying morbidity and mortality (21). 
In this study, we investigated the presence of chlamydiae in vascular tissues from 103 pet birds with atherosclerosis. The birds consisted mainly of parrots $(89.3 \%)$ with the highest incidences for African grey parrots and amazons, as described in other reports $(3,4)$. Atherosclerosis was often an ancillary finding. However atherosclerosis was the only disorder found in the majority of cases classified as grade 4. Histologic classification revealed no association between age of the birds and degree of atherosclerosis (Fig 1). This is in contrast to a study showing that severity of atherosclerosis in parrots increased with age (3). However, in our study smaller parrots with lower expectation of life - like cockatiels and lovebirds with severe atherosclerosis were also investigated. On the other hand, none of the macaws that can reach high ages, showed severe atherosclerosis, as reported by Bavelaar and Beynen (3).

DNA degradation in formalin-fixed and paraffin-embedded sections was one of the problems in our study - the older the samples, the larger the chance of encountering a fragmented DNA (21). Our attempts with primer pair 16SF2 (5'-CCGCCCGTCACATCATGG-3') and 23R (5'-TACTAAGATGTTTCAGTTC-3') to amplify a 585bp-product of the $16 \mathrm{~S} / 23 \mathrm{~S}$ rRNA intergenic spacer region of chlamydiae were not successful (unpublished data). Therefore it was important to design a PCR assay that amplified a short DNA fragment for the detection of chlamydiae in our samples. Only 4 of 103 birds - two amazons, one budgerigar and one jacana - tested with a 23s rRNA PCR that amplifies a 92bp fragment were positive. With online BLAST searches a high similarity to $C p$. psittaci for the two amazons and the jacana was found. No further identification was possible with the budgerigar sample. This may be due to a new species or a species, which was not described as yet.

Immunohistochemistry was not to our satisfaction. None of the 9 IHC-positive sections could be confirmed by repeated PCR run. Furthermore, sections from the 4 PCR positive cases were negative in IHC. Poor correlation between PCR and IHC in atherosclerotic tissue has been reported in other studies $(6,16)$. Of major concern are non-specific staining reactions of the chlamydial antibodies with cell components in the atherosclerotic plaque. Hoymans et al. could show a high affinity of the chlamydial antibodies to oxidized low-density lipoproteins in atherosclerotic lesions, a phenomenon of molecular mimicry that leads to false-positive results (16). Another explanation could be that chlamydia antigens rather than viable bacteria persist in the specimens, as described by Meijer et al. (20). However our study confirms the observation of Hoymans et al. as even negative control sections demonstrated non-specific staining reactions. Furthermore false negative IHC-results could be ascribed to the fact that the small sections tested did not contain any chlamydial antigen. 
Interestingly, the two amazons sequenced positive for $C p$. psittaci came from $C p$. psittaciinfected populations and had been tested for chlamydia antigen at post mortem with the Chlamydia-antigen ELISA (IDEIA ${ }^{\mathrm{TM}}$ Chlamydia ref K6002, DakoCytomation, Denmark). It was performed on liver, spleen and cloacal samples with a negative result in both birds. The PCR, which is more sensitive, suggests that the level of chlamydial antigen was too low to be detected in the ELISA.

An etiological role of chlamydiae can't be confirmed by our study. If chlamydiae cause atherosclerosis in birds, their presence would be expected to precede that of disease. One hypothesis states chlamydiae to act as focal point in atherosclerosis. After initiation of the disease, they disappear from the lesions. In this case one would expect to find chlamydiae with a higher prevalence in slight atherosclerotic lesions rather than in the severe ones. Furthermore it is still possible that after initiating the disease process the organism has disapperared and thus is not detectable when atherosclerosis develops. However experimental infections with different $C p$. psittaci strains in birds showed, that no signs of atherosclerosis were found at necropsy, even 210 days post infectionem $(7,18)$. In another hypothesis chlamydiae initiate atherosclerosis and persist in vascular tissue. Thus their prevalence would be similar in every grade of atherosclerosis. If chlamydiae play no role in atherosclerosis, but prefer the altered vascular tissue as a favorable environment for persistence or growth, one would expect to detect them mainly in lesions of grade 3 or 4 . In our study chlamydiae were only found in older lesions (grade 3 or grade 4), suggesting no role for them as initiators of atherosclerosis.

In conclusion, we could detect chlamydiae in atherosclerotic tissue from 4 of 103 birds. The low incidence $(3.9 \%)$, the occurrence only in advanced stages of atherosclerosis and the association with Cp. psittaci-infected avian populations, leads us to the conclusion, that there is probably no association between chlamydiae and atherosclerosis in pet birds. 


\section{REFERENCES}

1. Albicker-Rippinger P., and R. K. Hoop. Krankheitsursachen bei Papageienvögeln (Psittaciformes) und Sperlingsvögeln (Passeriformes). Tierärztl Prax 27 (K):245-254. 1999.

2. Apfalter, P., W. Barousch, M. Nehr, B. Willinger, M. Rotter, and A. M. Hirschl. No evidence of involvement of Chlamydia pneumoniae in severe cerebrovascular atherosclerosis by means of quantitative real-time polymerase chain reaction. Stroke 35:2024-2028. 2004.

3. Bavelaar, F. J., and A. C. Beynen. Severity of atherosclerosis in parrots in relation to the intake of alpha-linolenic acid. Avian Dis 47:566-577. 2003.

4. Bavelaar, F. J., and A. C. Beynen. Atherosclerosis in parrots. A review. Vet Q 26:5060. 2004.

5. Blasi, F., F. Denti, M. Erba, R. Cosentini, R. Raccanelli, A. Rinaldi, L. Fagetti, G. Esposito, U. Ruberti, and L. Allegra. Detection of Chlamydia pneumoniae but not Helicobacter pylori in atherosclerotic plaques of aortic aneurysms. J Clin Microbiol 34:27662769. 1996.

6. Boman, J., and M. R. Hammerschlag. Chlamydia pneumoniae and atherosclerosis: critical assessment of diagnostic methods and relevance to treatment studies. Clin Microbiol Rev 15:1-20. 2002.

7. Buuren, van C. E., G. M. Dorrestein, and J. E. van Dijk. Chlamydia psittaci infections in birds: a review on the pathogenesis and histopathological features. Vet Q 16:38-41. 1994.

8. Cochrane, M., A. Pospischil, P. Walker, H. Gibbs, and P. Timms. Distribution of Chlamydia pneumoniae DNA in atherosclerotic carotid arteries: significance for sampling procedures. J Clin Microbiol 41:1454-1457. 2003.

9. Danesh, J., R. Collins, and R. Peto. Chronic infections and coronary heart disease: is there a link?[see comment]. Lancet 350:430-436. 1997.

10. Everett, K. D. Chlamydia and Chlamydiales: more than meets the eye. Vet Microbiol 75:109-126. 2000.

11. Everett, K. D., R. M. Bush, and A. A. Andersen. Emended description of the order Chlamydiales, proposal of Parachlamydiaceae fam. nov. and Simkaniaceae fam. nov., each containing one monotypic genus, revised taxonomy of the family Chlamydiaceae, including a new genus and five new species, and standards for the identification of organisms. Int J Syst Bacteriol 49 Pt 2:415-440. 1999. 
12. Fabricant, C. G. Atherosclerosis: the consequence of infection with a herpesvirus. Adv Vet Sci Comp Med 30:39-66. 1985.

13. Fabricant, C. G., and J. Fabricant. Atherosclerosis induced by infection with Marek's disease herpesvirus in chickens. Am Heart J 138:S465-468. 1999.

14. Farsak, B., A. Yildirir, Y. Akyon, A. Pinar, M. Oc, E. Boke, S. Kes, and L. Tokgozoglu. Detection of Chlamydia pneumoniae and Helicobacter pylori DNA in human atherosclerotic plaques by PCR. J Clin Microbiol 38:4408-4411. 2000.

15. Grayston, J. T. Infections caused by Chlamydia pneumoniae strain TWAR.[see comment]. Clinical Infectious Diseases 15:757-761. 1992.

16. Hoymans, V. Y., J. M. Bosmans, D. Ursi, W. Martinet, F. L. Wuyts, E. Van Marck, M. Altwegg, C. J. Vrints, and M. M. Ieven. Immunohistostaining assays for detection of Chlamydia pneumoniae in atherosclerotic arteries indicate cross-reactions with nonchlamydial plaque constituents. J Clin Microbiol 42:3219-3224. 2004.

17. Ieven, M. M., and V. Y. Hoymans. Involvement of Chlamydia pneumoniae in atherosclerosis: more evidence for lack of evidence. J Clin Microbiol 43:19-24. 2005.

18. Jakoby, J. R., and H. Gerbermann. Experimentelle Chlamydialinfektion bei Amazonen. Der praktische Tierarzt 5:453 - 458. 1982.

19. Johnson, J. H., D. N. Phalen, V. H. Kondik, T. Tippit, and D. L. Graham. Atherosclerosis in psittacine birds. Proceedings Association of Avian Veterinarians:87-93. 1992.

20. Meijer, A., P. J. Roholl, S. K. Gielis-Proper, and J. M. Ossewaarde. Chlamydia pneumoniae antigens, rather than viable bacteria, persist in atherosclerotic lesions. J Clin Pathol 53:911-916. 2000.

21. Mygind, T., S. Birkelund, E. Falk, and G. Christiansen. Evaluation of real-time quantitative PCR for identification and quantification of Chlamydia pneumoniae by comparison with immunohistochemistry. J Microbiol Methods 46:241-251. 2001.

22. Saikku, P., M. Leinonen, K. Mattila, M. R. Ekman, M. S. Nieminen, P. H. Makela, J. K. Huttunen, and V. Valtonen. Serological evidence of an association of a novel Chlamydia, TWAR, with chronic coronary heart disease and acute myocardial infarction. Lancet 2:983986. 1988.

23. Shi, Y., and O. Tokunaga. Chlamydia pneumoniae and multiple infections in the aorta contribute to atherosclerosis. Pathol Int 52:755-763. 2002. 
24. Soldati, G., Z. H. Lu, L. Vaughan, A. Polkinghorne, D. R. Zimmermann, J. B. Huder, and A. Pospischil. Detection of mycobacteria and chlamydiae in granulomatous inflammation of reptiles: a retrospective study. Vet Pathol 41:388-397. 2004.

25. Sudler, C., L. E. Hoelzle, I. Schiller, and R. K. Hoop. Molecular characterisation of chlamydial isolates from birds. Vet Microbiol 98:235-241. 2004.

\section{ACKNOWLEDGMENTS}

We are grateful to Madeleine Bart, Corinne Rutz and Christine Sudler (Institute of Veterinary Bacteriology, NRGK, University of Zurich), Ludwig Hölzle (Institute of Veterinary Bacteriology, University of Zurich), Taurai Tasara (Institute for Food Safety and Hygiene, University of Zurich), Nicole Borel, Komkrich Teankum, Roseline Weilenmann and Sabina Wunderlin (Institute of Veterinary Pathology, University of Zurich) for their help and support. 
Table 1. Avian orders and age of collected birds

\begin{tabular}{lccccccc}
\hline Avian order & Total & $\begin{array}{c}\text { Age } \\
\text { (years) } \\
1 \text { to } 5\end{array}$ & 6 to 10 & 11 to 20 & 21 to 30 & $>30$ & Unknown \\
& \multicolumn{2}{c}{10} & 12 & 15 & 9 & 10 & 36 \\
\hline Psittaciformes & 92 & 10 & 1 & 0 & 0 & 0 & 1 \\
Passeriformes & 3 & 1 & 0 & 0 & 0 & 0 & 1 \\
Anseriformes & 2 & 1 & 0 & 0 & 0 & 0 & 2 \\
Ciconiiformes & 2 & 0 & 1 & 1 & 0 & 0 & 2 \\
Others & 4 & 0 & 14 & 16 & 9 & 10 & 42 \\
Total & 103 & 12 & &
\end{tabular}

Table 2. Results of histopathology, immunohistochemistry (IHC) and sequence analysis of the 4 PCR positive cases.

\begin{tabular}{lcccc}
\hline Bird & $\begin{array}{c}\text { Grade of } \\
\text { atherosclerosis }\end{array}$ & IHC & PCR & Sequencing \\
\hline Amazon & 4 & - & + & $C p \cdot$ psittaci \\
Budgerigar & ne & - & + & $C p \cdot$ species \\
Amazon & 3 & - & + & $C p \cdot$ psittaci \\
Jacana & 4 & - & + & $C p \cdot$ psittaci \\
\hline
\end{tabular}

ne: not evaluated 
Fig. 1. Distribution of the degree of atherosclerosis among the different age groups (only 56 psittacines with known age are represented)

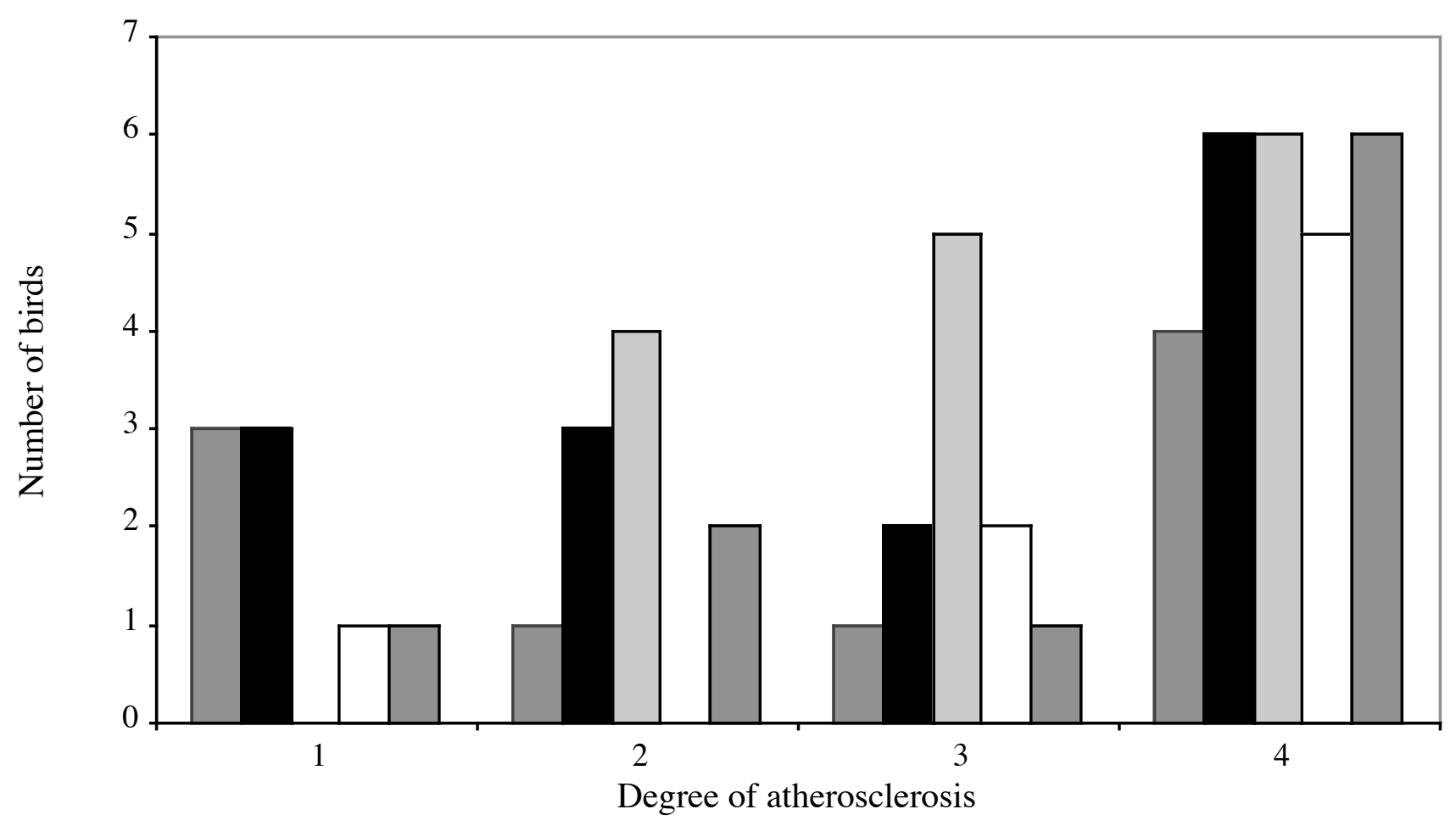

\begin{tabular}{|c|c|c|c|c|}
\hline$\square 1$ to 5 & $\mathbf{\square} 6$ to 10 & 11 to 20 & 口 21 to 30 & $\square>30$ \\
\hline
\end{tabular}


Fig. 2. Distribution of the degree of atherosclerosis among the different species

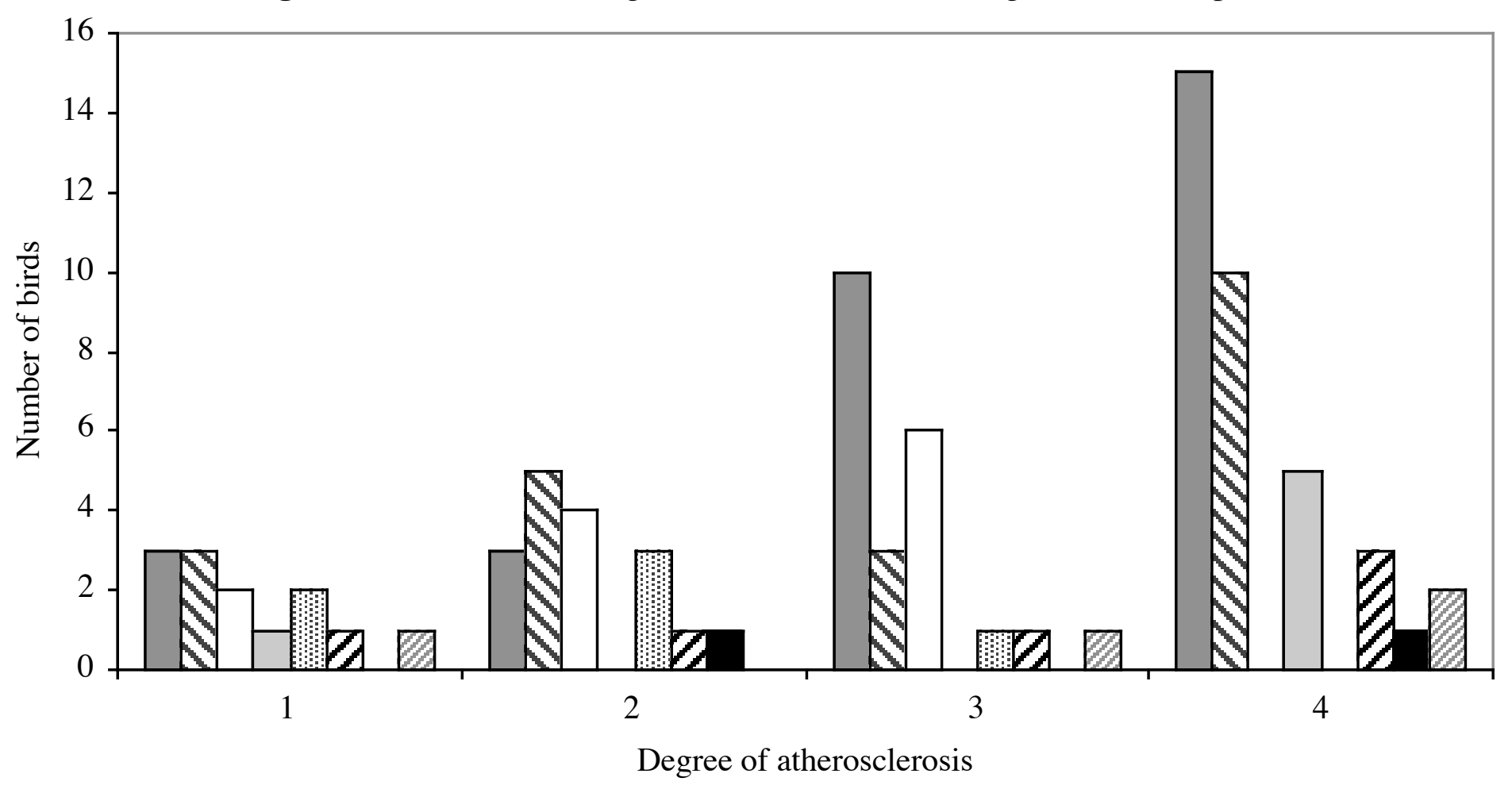

\begin{tabular}{|c|c|c|c|}
\hline African grey & ๑ Amazon & 口Macaw & 口Lovebird \\
\hline 日Budgerigar & هCockatiel & - Cockatoo & 口Others \\
\hline
\end{tabular}



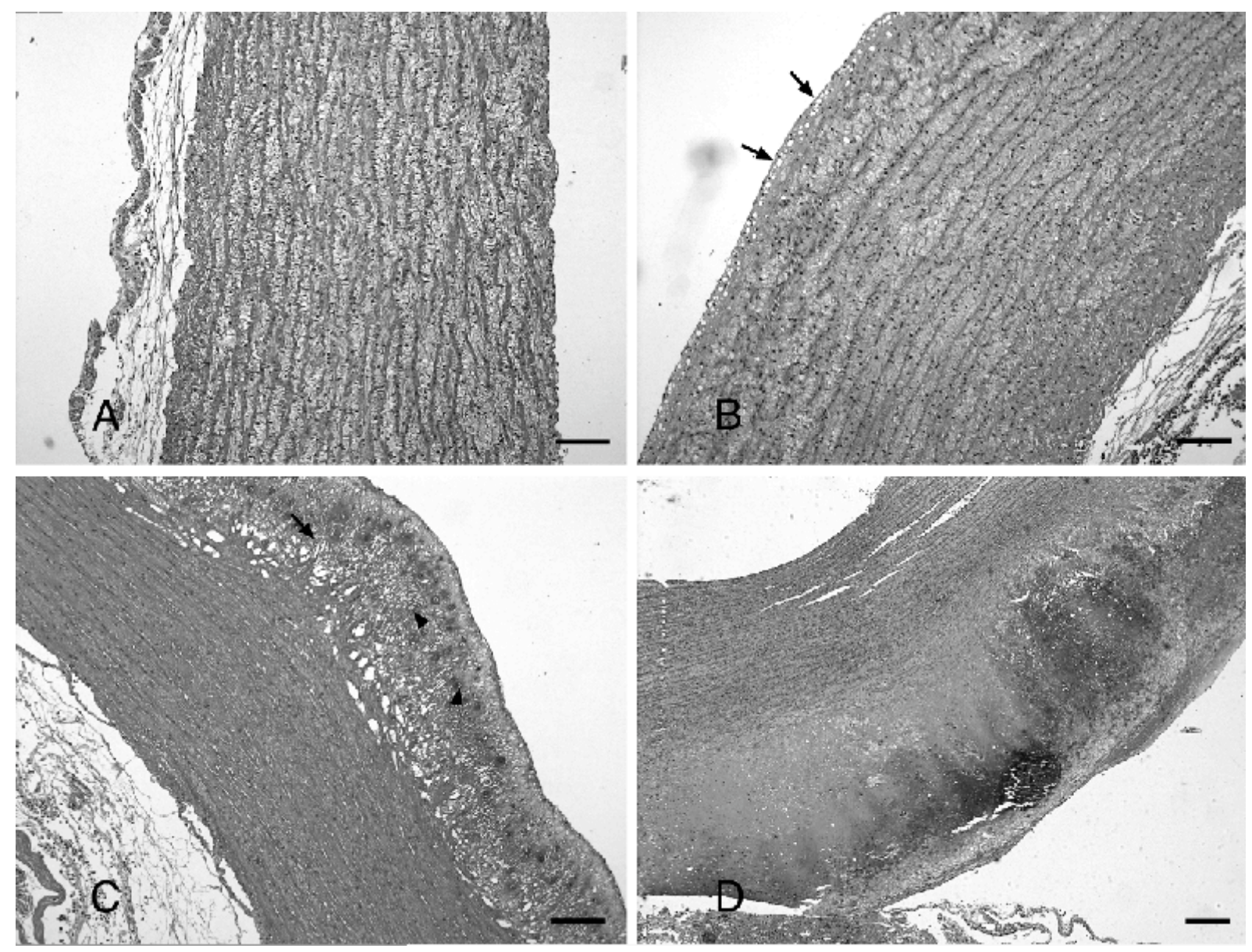

Fig. 3. The extent of atherosclerosis in 4 grades. (A) Grade $1=$ slight atherosclerosis with fragmentation of the elastic fibres and increase of the extracellular matrix in the intima and media. Bar $=100 \mu \mathrm{m}$. (B) Grade $2=$ moderate atherosclerosis with additional accumulation of lipid vacuoles (arrows) in the intima. Bar $=100 \mu \mathrm{m}$. (C) Grade $3=$ development of plaques consisting of lipids, cholesterol clefts (arrow), fibrous connective tissue and chondrocytes (arrowheads). Bar $=100 \mu \mathrm{m}$. (D) Grade $4=$ severe atherosclerosis. Bar $=200 \mu \mathrm{m}$. 


\section{Lebenslauf}

Personalien

$\begin{array}{ll}\text { Name } & \text { Olivier Andreas Schenker } \\ \text { Geburtsdatum } & 02.02 .1973 \\ \text { Geburtsort } & \text { Genf } \\ \text { Nationalität } & \text { Schweizer } \\ \text { Heimatort } & \text { Walterswil SO }\end{array}$

\section{Ausbildung}

$1980-1985$

$1985-1989$

Primarschule Lenzburg

$1989-1993$

Bezirksschule Lenzburg

$1993-1997$

Matura Typus C, Kantonsschule Aarau

$1998-2000$

8 Semester Biologie an der Universität Zürich

2004

Studium der Veterinärmedizin an der Universität Zürich

$2004-2006$

Staatsexamen als Tierarzt

Dissertation am Institut für Veterinärbakteriologie der Universität Zürich

Zürich, 25. August 2006 